\title{
Changes in Growth and Photosynthetic Parameters and Medicinal Compounds in Eleutherococcus senticosus Harms under Drought Stress
}

\author{
Mingyuan Xu and Yingwei Wang \\ First Affiliated Hospital, Heilongjiang University of Chinese Medicine, \\ Harbin 150040, China; and Heilongjiang University of Chinese Medicine, \\ Harbin 150040, China
}

\section{Qianbo Wang}

The First Affiliated Hospital of Clinical Medicine of Guangdong Pharmaceutical University, Guangzhou 510000, China

\author{
Shenglei Guo \\ Heilongjiang University of Chinese Medicine, Harbin 150040, China; and \\ HeiLongJiang ZBD Pharmaceutical Co., Ltd, Harbin 150060, China
}

Yang Liu, Jia Liu, and Zhonghua Tang

Key Laboratory of Plant Ecology, Northeast Forestry University, Harbin 150040, China

\section{Zhenyue Wang Heilongjiang University of Chinese Medicine, Harbin 150040, China}

Additional index words. drought stress, Eleutherococcus senticosus, multiple reaction monitoring, quantitative analysis, UPLC-electrospray ionization-MS

\begin{abstract}
In this investigation, changes in growth and photosynthetic parameters were used to explain the effects of drought stress on morphology and photosynthesis of Eleutherococcus senticosus. Liquid chromatography (LC)-mass spectroscopy (MS) was used to determine the content of eleutheroside $B$, eleutheroside $E$, isofraxidin, hyperoside, rutin, and kaempferol under different drought stress conditions to explain the effects of drought stress on secondary metabolism of Eleuthero. Growth and photosynthetic physiological parameters showed that drought stress could inhibit the growth and photosynthesis of Eleuthero. The compounds studied showed the same cumulative trend in various organs of Eleuthero under different drought stress conditions, with the highest content in the moderate drought stress group and the lowest in the severe drought stress group. Among them, the content of eleutheroside $B$ was found to be higher in the 5-yearold stem. The content of eleutheroside $E$ was higher in the 3-year root. The content of isofraxidin was highest in the 5-year-old root. The content of hyperoside, rutin, and kaempferol were higher in the 3-year-old leaves. The results show that a wet soil environment was beneficial to growth and photosynthesis of Eleutherococcus senticosus, and moderate drought stress is conducive to the accumulation of its active ingredients.
\end{abstract}

Eleutherococcus senticosus (Ruper. et Maxim.) Harms is a species of a small woody shrub that belongs to the family Araliaceae (Huang et al., 2011). It is commonly used in

Received for publication 24 July 2018. Accepted for publication 23 Sept. 2019.

We are grateful for the financial support of the National Key Research and Development Program of China (No. 2016YFC0500303); Heilongjiang Province Foundation for the National Key Research and Development Program of China (No. GX17C006); the Post-Doctoral Foundation of Heilongiiang Province, China (LBH-Z17208); and the Key Project of Heilongjiang Provincial Administration of Traditional Chinese Medicine (No. 2018-009).

Z.-Y.W. is the corresponding author. E-mail: Wangzhen yue@163.com.
2015) and encourages immunomodulation; thus, it has been widely used to treat chronic bronchitis, neurasthenia, hypertension, and ischemic heart disease (Sun et al., 2016). The components of phenolic, triterpenoid saponins, lignan, coumarins, flavones, polysaccharides, and volatility have been detected in the Eleuthero (Huang et al., 2011; Jiang et al., 2006; Li et al., 2016).

The chemical compounds of Eleuthero raw materials used in this study are phenolic compounds. Phenols are the most widely distributed metabolites involved in interactions between biology and the environment (Garcia-Calderon et al., 2015). The accumulation of phenols may also affect other secondary metabolite pathways, including alkaloid pathways, because plant defense is a complicated system (Ferreres et al., 2008; Mustafa and Verpoorte, 2007). Phenolic compounds and related pathways can be influenced by exposure of plants to abiotic stresses, such as adverse environmental conditions (Dixon and Paiva 1995; Harb et al., 2010). However, plants have evolved to survive harsh conditions, for example, by using their metabolic capacity to produce a variety of secondary metabolites. Eleutheroside $\mathrm{B}$, eleutheroside $\mathrm{E}$, isofraxidin, hyperoside, rutin, and kaempferol are the main phenols in Eleuthero (Baczek et al., 2017; Yang et al., 2012, 2013). Eleutheroside B and $\mathrm{E}$ are lignans (Lee et al., 2004), isofraxidin is a coumarin compound (Yamazaki and Tokiwa, 2010), and hyperoside, rutin, and kaempferol belong to the flavonoid group (Baczzek et al., 2017). These compounds are derived from shikimic acid (Gamir et al., 2014; Schafellner et al., 1999), the contents of which also seem to increase from water deficit (Becerra-Moreno et al., 2015).

To the best of our knowledge, there is no comprehensive study on the effects of drought stress on Eleuthero. The present study focused on photosynthetic physiological parameters and targeted analysis of metabolite features of roots, stems, and leaves under different water treatments. Collectively, these data will enable assessment of the difference in growth, photosynthetic physiological responses, and secondary metabolism responses to water restriction throughout the plant.

\section{Materials and Methods}

Russia (called Siberian ginseng). The roots and stems of the plant are recognized as a tonic herb that has a ginseng-like effect (Bucci, 2000). Eleuthero root is considered a pharmacopeia raw material in many countries (Europe, United States, Japan). Eleuthero invigorates qi, strengthens the spleen, and nourishes the kidney (Han et al., 2014). Thus, Eleuthero may be used for yang deficiency of the spleen and kidney, body weakness and hyperdynamics, poor appetite, aches of the waist and knee, insomnia (Han et al., 2017). Eleuthero has anti-inflammatory (Fei et al., 2014; Jiang and Wang, 2015), antioxidant (Kim et al., 2015), antifatigue (Huang et al., 2011; Jiang and Wang, 2015), and anticancer properties (Cichello et al.,
Chemicals and reagents. Eleutheroside B, eleutheroside $\mathrm{E}$, isofraxidin, hyperoside, rutin, and kaempferol were purchased from the Chinese National Institute of Control of Pharmaceutical and Biological Products (Beijing, China). Water used for ultra performance LC-tandem MS (UPLC-MS) analysis was prepared with a Milli-Q water purification system bought from Millipore (Milford, MA). Acetonitrile (J \& K Scientific Ltd., Beijing, China) was high-performance LC grade. All other chemicals used in the method were of analytical grade.

Plant materials. Seedlings of 3-year-old Eleuthero were obtained from Qitaihe, Heilongjiang Province, China (geographic coordinates: lat. $45^{\circ} 95^{\prime} \mathrm{N}$, long. $131^{\circ} 05^{\prime} \mathrm{E}$ ) and 


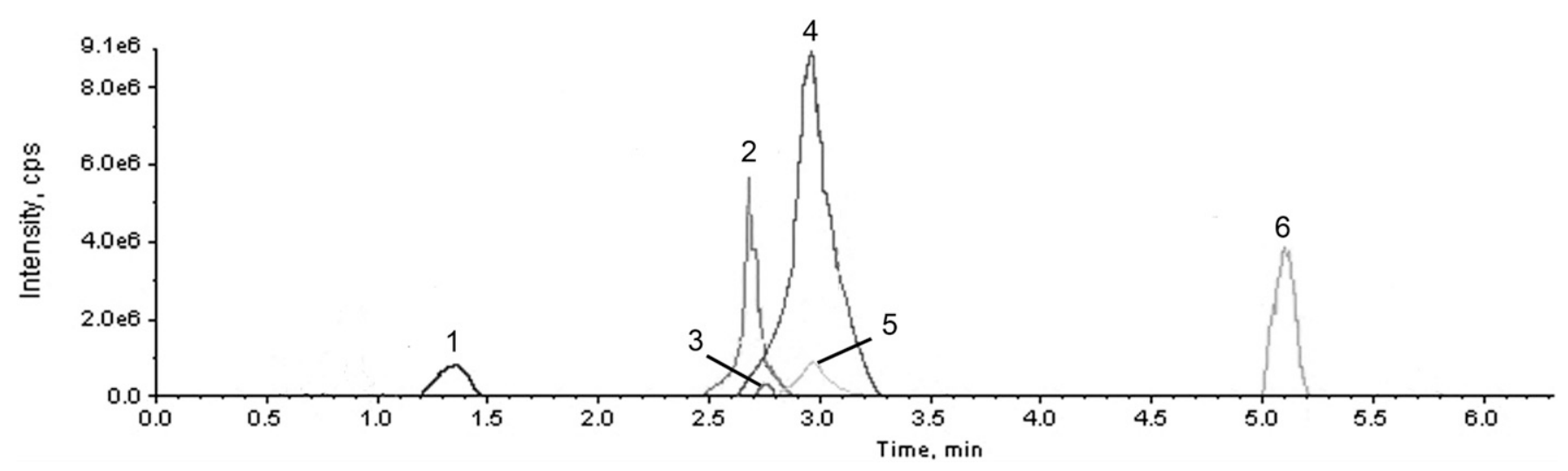

Fig. 1. Multiple reaction monitoring chromatograms of the objective compounds. (1) Eleutheroside B, (2) eleutheroside E, (3) isofraxidin, (4) hyperoside, (5) rutin, and (6) kaempferol.

planted in the Heilongjiang University of Chinese Medicine Botanical Garden, Harbin, Heilongjiang Province, China (geographic coordinates: lat. $45^{\circ} 72^{\prime} \mathrm{N}$, long. $\left.126^{\circ} 64^{\prime} \mathrm{E}\right)$ in February. A month later (March), seedlings were transplanted into $30-\mathrm{cm}$ diameter pots containing a mixture of garden soil and vermicomposting $(3: 1, \mathrm{w} / \mathrm{w})$; soil $\mathrm{pH}$ was 6.50 . The experiment was conducted in a glasshouse with temperature ranging from 20 to $30{ }^{\circ} \mathrm{C}$ under natural light conditions (day length: $13 \mathrm{~h}$ ). The climate of Harbin is temperate continental monsoon type. The whole plant was dissected to obtain roots, stems, and leaves for metabolic analysis. The raw materials were dried at $60{ }^{\circ} \mathrm{C}$ for $48 \mathrm{~h}$. The experiment was performed in three replications.

Drought stress treatment. One month after transplantation (April), the control $(\mathrm{GK})$, moderate $\left(\mathrm{W}_{1}\right)$, and severe $\left(\mathrm{W}_{2}\right)$ drought stress treatment was started. The soil moisture of $\mathrm{GK}, \mathrm{W}_{1}$, and $\mathrm{W}_{2}$ was maintained at 0.8 to $0.9 \mathrm{~g} / \mathrm{g}, 0.5 \pm 0.05 \mathrm{~g} / \mathrm{g}$, and $0.3 \mathrm{~g} \pm$ $0.05 \mathrm{~g} / \mathrm{g}$, respectively. The moisture in pot soil was evaluated regularly by measuring the soil water content percentage: $1 \mathrm{~g}$ soil from pots of each treatment, as well as the control, was taken, oven-dried, and weight was taken again. Soil water content percentage was calculated by using the formula:

$$
\begin{aligned}
& \text { Soil water content percentage } \\
& =\frac{\text { Fresh soil weight }- \text { dry soil weight }}{\text { fresh soil weight }} \times 100
\end{aligned}
$$

This measurement was repeated regularly at 1-d intervals, and water $(100-400 \mathrm{~mL})$ was supplied to pots of each treatment during plant development. There were three replicates in each experimental group, and six seedlings in each replicate. The experiment was conducted throughout plant developmental stages (from April to June).

Determination of growth parameters. A sampling of plant material was done after 2 months of drought stress treatment. Six plants were randomly selected in each treatment group; measurements of total leaf area (TLA), plant height (PH), and leaf number (LN) were performed. TLA was measured with LI-3100 leaf area meter (LI-COR Bio-

Table 1. Delustering voltages, collision voltages, and collision chamber emission voltages of the medicinal compounds.

\begin{tabular}{llccc}
\hline Analyte & Ion pairs $(\mathrm{m} / \mathrm{z})$ & DP $(\mathrm{V})$ & $\mathrm{CE}(\mathrm{V})$ & $\mathrm{CXP}(\mathrm{V})$ \\
\hline Eleutheroside E & $765.3 \rightarrow 603.1$ & 70 & 62 & 23 \\
Rutin & $611.1 \rightarrow 464.8$ & 80 & 20 & 13 \\
Kaempferol & $287.1 \rightarrow 153.2$ & 70 & 51 & 10 \\
Isofraxidin & $223.1 \rightarrow 206.3$ & 60 & 40 & 9 \\
Eleutheroside B & $155.0 \rightarrow 92.9$ & 70 & 21 & 17 \\
Hyperoside & $395.1 \rightarrow 232.1$ & 70 & 40 & 17 \\
\hline
\end{tabular}

$\mathrm{DP}=$ delustering voltages, $\mathrm{CE}=$ collision voltages, $\mathrm{CXP}=$ collision chamber emission voltages.

sciences, Lincoln, NE), and PH was measured using digital calipers and a ruler.

Determination of photosynthetic parameters. Photosynthetic parameters were measured using a portable photosynthesis system (Li-6400XT, LI-COR) in a glasshouse from 10:00 AM to 1:00 PM on 30 June 2017. Weather was normal during the investigation. Six plants were randomly selected in each treatment group, and the net photosynthetic rate $\left(\mathrm{P}_{\mathrm{n}}\right)$, stomatal conductance $\left(g_{\mathrm{S}}\right)$, transpiration rate $(\mathrm{E})$, and intercellular $\mathrm{CO}_{2}$ concentration $(\mathrm{Ci})$ values were read directly using the photosynthesis system.

$L C-M S$ analysis of the active medicinal ingredient. Dried leaf, stem, and root of Eleuthero were ground in a mill and passed through a 35-mesh sieve. Approximately $2 \mathrm{~g}$ of dry powdered plant material was extracted with $10 \mathrm{~mL}$ of methanol $(80 \%)$ by reflux for $45 \mathrm{~min}$. The extract was repeatedly filtered, and the filtrate was collected. The extract was subjected to centrifugation at $14,000 \mathrm{rpm}$ at $4{ }^{\circ} \mathrm{C}$ for $10 \mathrm{~min}$. The supernatant was removed, and the extract was concentrated by evaporation under a vacuum to dryness. Then the precipitate was dissolved with methanol to a volume of $1.0 \mathrm{~mL}$. All samples were filtered through a $0.22-\mu \mathrm{m}$ diameter micropore filter membrane, which could be directly injected for LC-MS analysis.

The analysis of compounds is described in our previous studies (Wu et al., 2018); roots and stems were pulverized by using a grinding instrument (MM 400, Retsch, Haan, Germany), and 50-mg tissue aliquots were extracted with $1.0 \mathrm{~mL} 70 \%$ aqueous methanol containing $0.1 \mathrm{mg} / \mathrm{L}$ lidocaine for watersoluble metabolites at $4{ }^{\circ} \mathrm{C}$ overnight and vortexed three times. The extracts were clarified by centrifugation, combined, evaporated, and then filtered through $0.45-\mu \mathrm{m}$ nylon membranes (SCAA 104; ANPEL http://www. anpel.com.cn) before LC-MS analysis.

UPLC-MS analysis was performed with a Waters ACQUITY UPLC system (Waters Corporation, Tokyo, Japan) coupled to an LC-20AD pump, SIL-20A autosampler (Waters Corporation). The ACQUITY-UPLC BEH C18 column $(1.7 \mu \mathrm{m}, 2.1 \mathrm{~mm} \times 50 \mathrm{~mm})$ used for UPLC analysis was held at $25^{\circ} \mathrm{C}$; injection volume was $10.0 \mu \mathrm{L}$, and the flow rate was 0.5 $\mathrm{mL} / \mathrm{min}$. Mobile phase A comprised methanol, and mobile phase $\mathrm{B}$ comprised water. The column was eluted with a linear gradient of $25 \%$ A for 0 to $1.5 \mathrm{~min}, 25 \%$ to $50 \%$ A for 1.5 to $2.0 \mathrm{~min}, 50 \%$ A for 2.0 to $4.0 \mathrm{~min}, 50 \%$ to $90 \%$ A for 4.0 to $4.5 \mathrm{~min}, 90 \%$ A for 4.0 to 4.5 $\mathrm{min}, 90 \%$ to $25 \%$ A for 5.5 to $6.0 \mathrm{~min}$, and $25 \%$ A for 6.0 to $7.0 \mathrm{~min}$. The chromatograms of six medicinal compounds under a multireaction monitoring mode are shown in Fig. 1.

MS detection was performed using a QTRAP 5500 (AB SCIEX, Boston, MA) equipped with an Electrospray Ionization source with the following operating parameters: cone voltage of $3 \mathrm{kV}$ and ion source atomization temperature of $500{ }^{\circ} \mathrm{C} ; 25 \mathrm{psi}$ atomizing gas and $20 \mathrm{psi}$ air curtain gas. The ion pair, cluster voltage, collision voltage, and collision chamber injection voltage of six active metabolites are shown in Table 1. Both MS and MS/MS data were determined in the positive mode, and data were used for multiple reaction monitoring. Secondary MS of six active pharmaceutical ingredients is shown in Fig. 2.

Statistical analysis. It subjected all results to the analysis of variance (ANOVA) to determine the significant differences between various levels of salt treatment times. If ANOVA was performed, Duncan's honestly significant 
difference (HSD) post hoc tests were conducted to determine the differences between individual treatments (SPSS 22.0; SPSS Inc., Chicago, IL).

\section{Results and Discussion}

The changes in growth parameters. Changes in plant growth parameters are planted responses to drought stress on external morphology (Pellegrino et al.,
2010). Changes in PH showed in Fig. 3A. The moderate stress group $\left(\mathrm{W}_{1}\right)$ and the severe stress group $\left(\mathrm{W}_{2}\right)$ showed apparent differences from the control group (GK). The plant height in $\mathrm{W}_{1}$ was $57 \%$ lower than $\mathrm{GK}$, and in the $\mathrm{W}_{2}$ group it was $67 \%$ lower than GK. The results of LN count is shown in Fig. 3B. Higher LN was found in GK. LN in $\mathrm{W}_{1}$ was $43.84 \%$ lower than GK, and in $\mathrm{W}_{2}$ it was $32.61 \%$ lower than GK. The change of LA was opposite that of LN
(Fig. 3C), it was the highest in $\mathrm{W}_{1}$ and the lowest in the GK.

The results showed that drought stress could significantly inhibit $\mathrm{PH}$ and $\mathrm{LN}$ of Eleuthero and increase its leaf area. Soil water shortage also affects plant growth in many ways. On one hand, plant somatic cell division and differentiation require water participation. When water is absent, the speed of division and differentiation slows down or even stops (Van der Weele et al.,

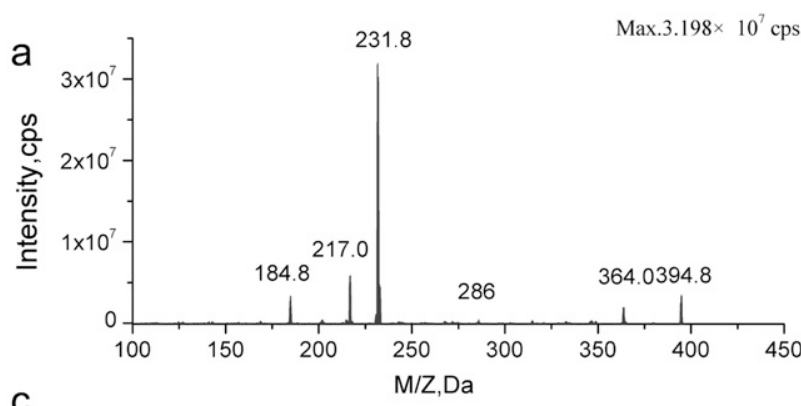

C
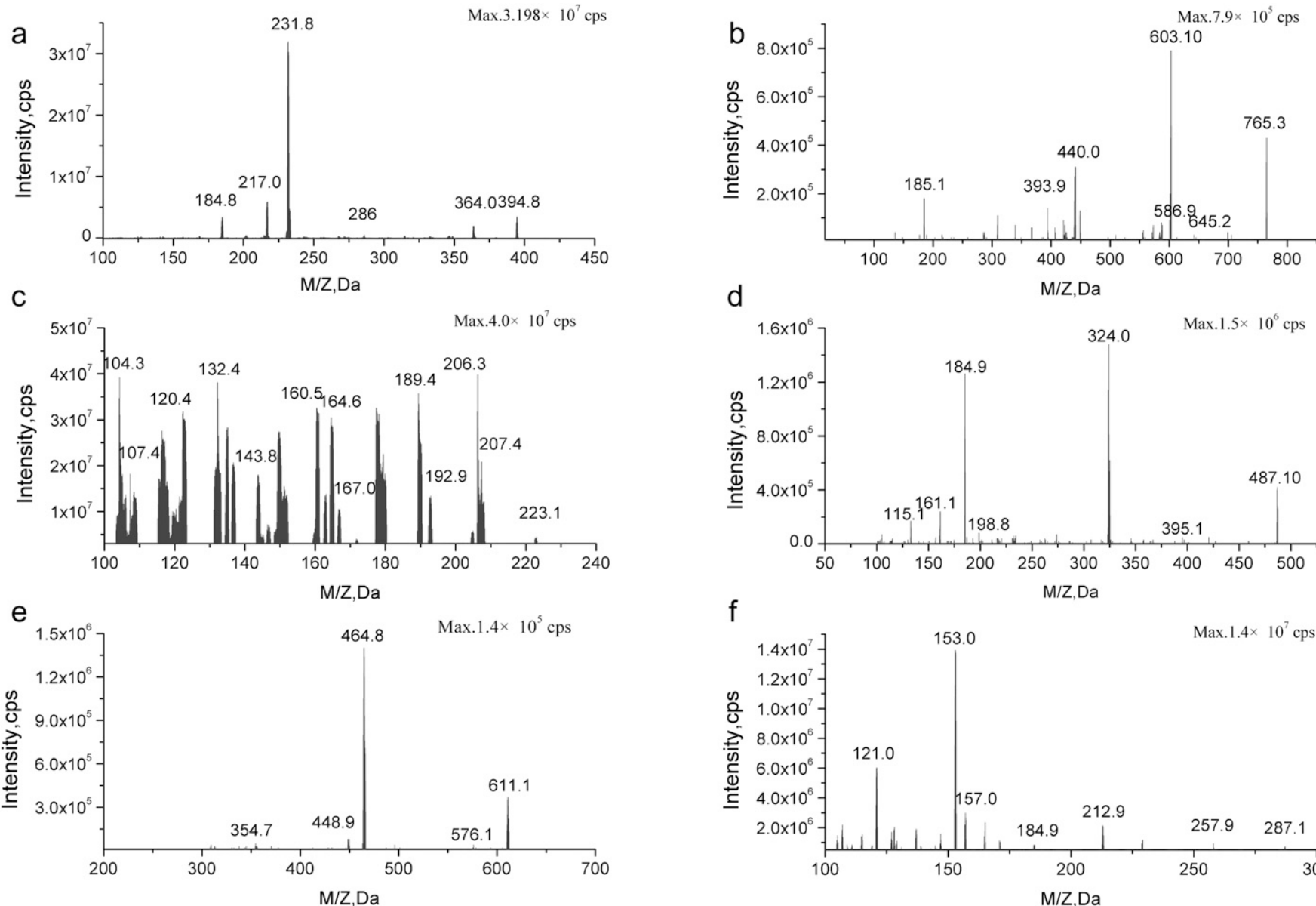

d

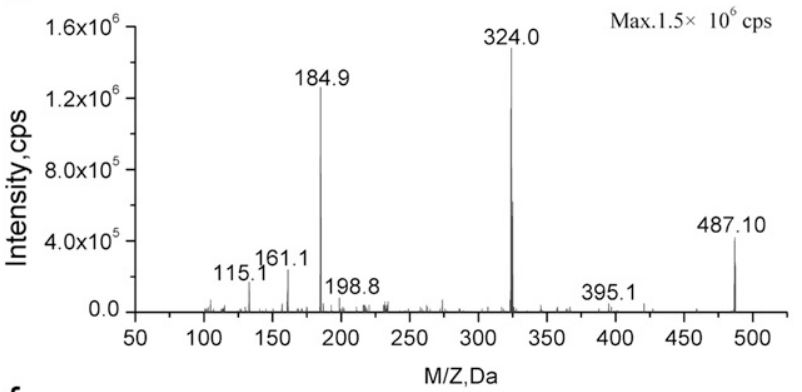

f

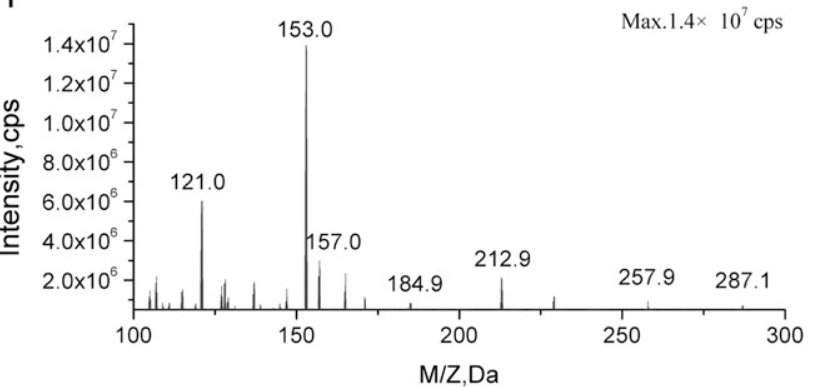

Fig. 2. Secondary mass spectrum of six active pharmaceutical ingredients. (1) Eleutheroside B, (2) eleutheroside E, (3) isofraxidin, (4) hyperoside, (5) rutin, and (6) kaempferol.
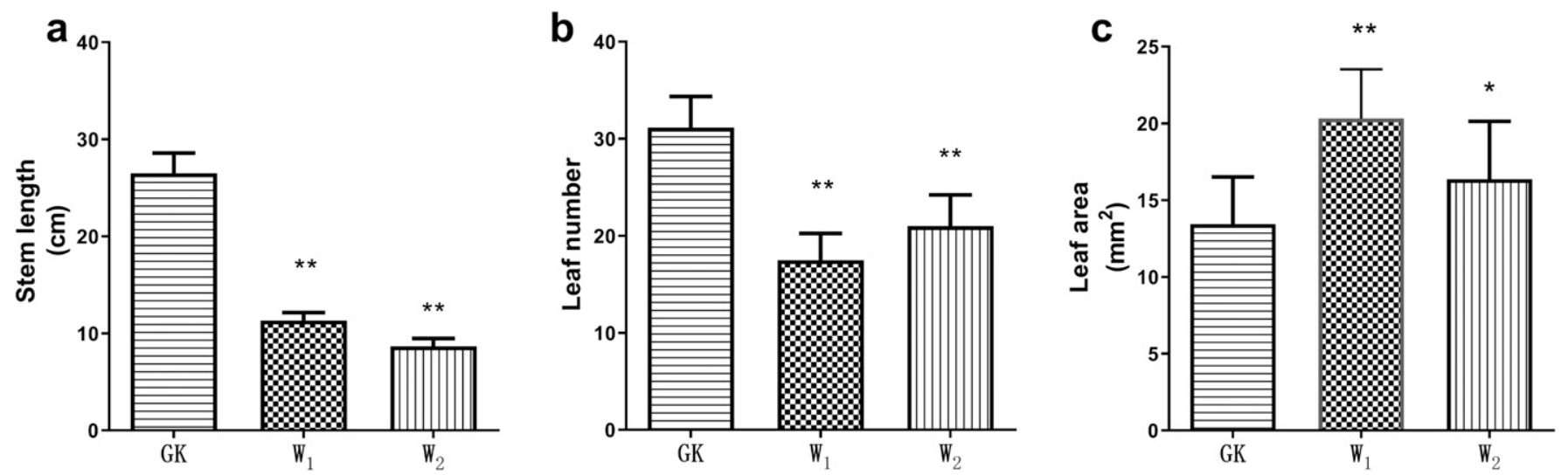

Fig. 3. Effect of water stress treatments on stem length, leaf number, and leaf area of Eleuthero. The soil moisture of the control (GK), moderate drought stress ( $\left.\mathrm{W}_{1}\right)$, and severe drought stress $\left(\mathrm{W}_{2}\right)$ groups was maintained at 0.8 to $0.9 \mathrm{~g} / \mathrm{g}, 0.5 \pm 0.05 \mathrm{~g} / \mathrm{g}$, and $0.3 \pm 0.05 \mathrm{~g} / \mathrm{g}$, respectively. $\mathrm{n}=6$. ${ }^{* *}$ Significant difference between the treatment group and the control group $(P<0.01)$; *significant difference between the treatment group and the control group $(P<0.05)$. 
A

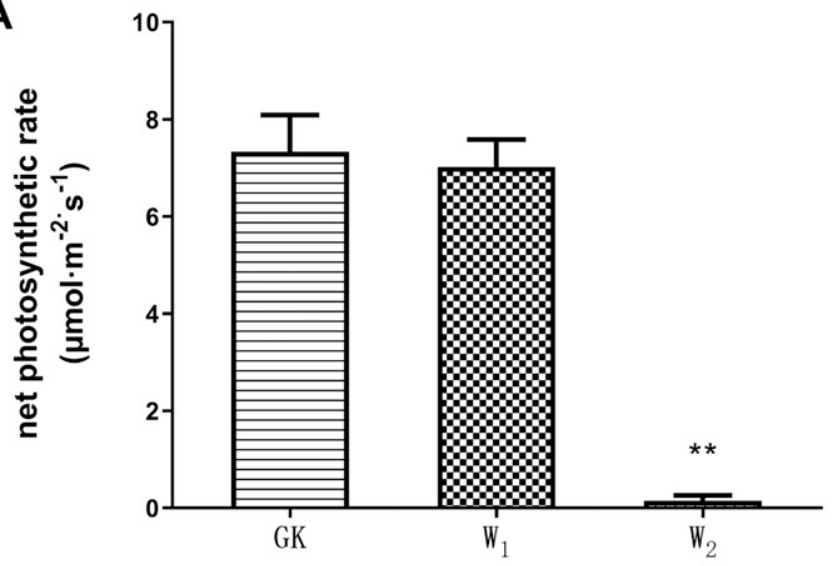

C

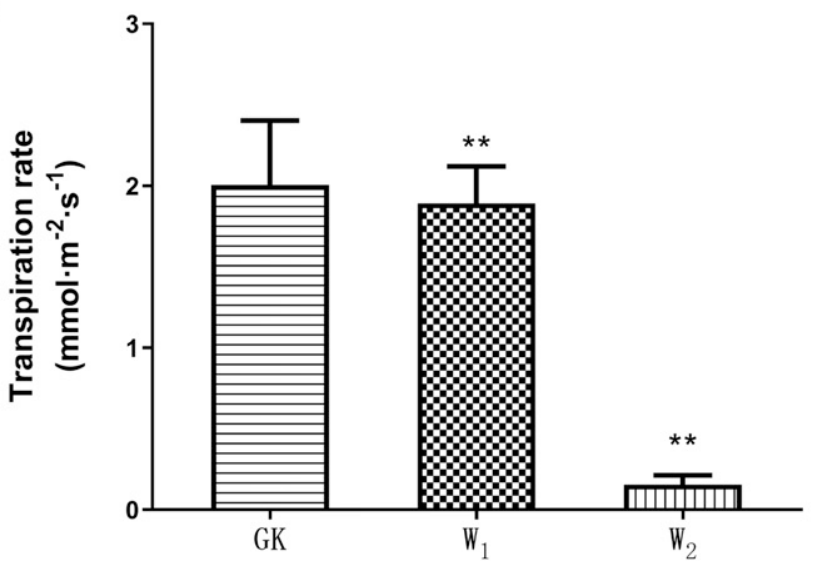

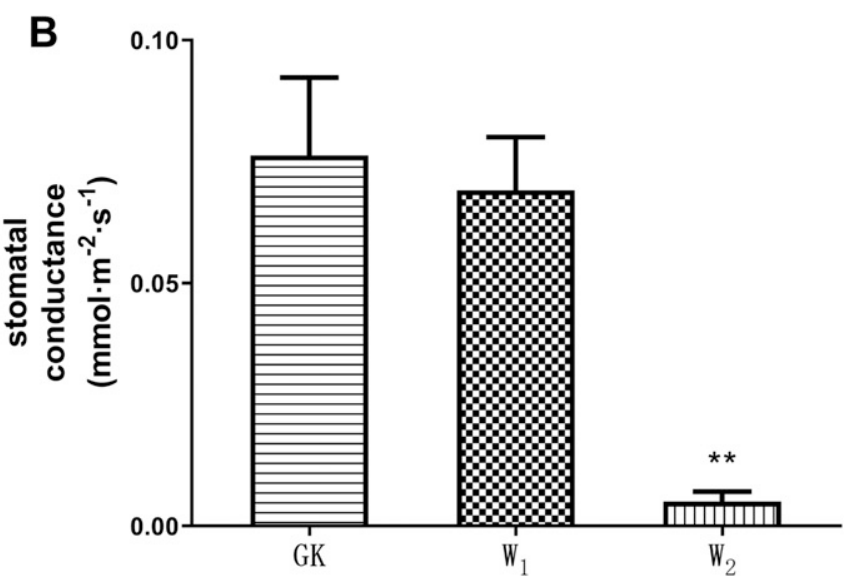

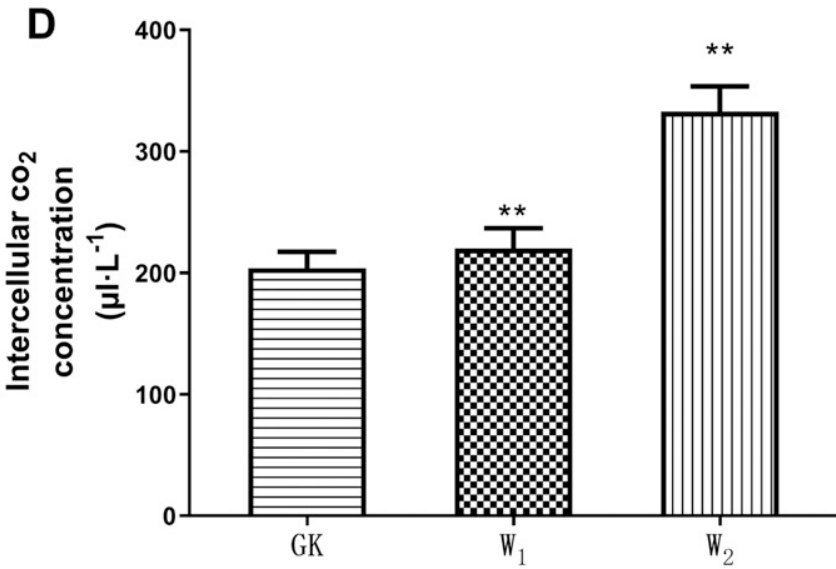

Fig. 4. Effect of water stress treatments on net photosynthetic rate, transpiration rate, stomatal conductance, and intercellular $\mathrm{CO}_{2}$ concentration of Eleuthero. The soil moisture of control $(\mathrm{GK})$, moderate drought stress $\left(\mathrm{W}_{1}\right)$, and severe drought stress $\left(\mathrm{W}_{2}\right)$ groups was maintained at 0.8 to $0.9 \mathrm{~g} / \mathrm{g}, 0.5 \pm 0.05 \mathrm{~g} / \mathrm{g}$, and $0.3 \pm$ $0.05 \mathrm{~g} / \mathrm{g}$ respectively. $\mathrm{n}=6$. **Significant difference between the treatment group and the control group $(P<0.01) ; *$ significant difference between the treatment group and the control group $(P<0.05)$.

2000). On the other hand, it affects the transport of substances in plants, and many substances in plants need water as a carrier (Walton et al., 1976). Under drought stress, the transport rate of substances slowed, and the assimilation products could not be readily distributed (Chaves, 1991). Also, drought affects plants' water absorption (Farooq et al., 2009). In this study, drought stress increased leaf area to a certain extent, which may be a response to adapt to drought conditions.

The changes in photosynthetic parameters. When Eleuthero was subjected to different drought stress treatments, significant differences in all photosynthetic parameters were observed. The $\mathrm{P}_{\mathrm{n}}$ and $g_{\mathrm{S}}$ decreased with the increase of drought stress (Fig. 4A and B). There was no significant difference between the GK and $\mathrm{W}_{1} \cdot \mathrm{P}_{\mathrm{n}}$ and $g_{\mathrm{S}}$ in $\mathrm{W}_{2}$ were significantly lower than $\mathrm{GK}$ and $\mathrm{W}_{1}$. The transpiration rate (E) was lower in the two drought stress groups (Fig. 4C), and it was lowest in $\mathrm{W}_{2}$. The concentration of intercellular carbon dioxide (Ci) showed the opposite trend (Fig. 4D). With worsening of drought stress, the two treatments were significantly higher than the control group, and the highest was in $\mathrm{W}_{2}$. This result showed that photosynthetic capability was best in leaves of Eleuthero cultivated under GK.
The factors leading to decline in photosynthetic rate include stomatal and nonstomatal limitations. The stomatal limiting factor was the decrease of $\mathrm{Ci}$ because of the decrease of the stomatal opening of leaves, whereas the nonstomatal factor was the decrease and accumulation of $\mathrm{CO}_{2}$ solubility, which resulted in the decrease of mesophyll photosynthetic capacity. Drought stress can inhibit photosynthesis in these two means (Berry and Downton, 1982). Previous studies (Colom and Vazzana, 2001; Rajendrudu et al., 2000) have shown that soil moisture is the main limiting factor for plant photosynthetic parameters. Moderate water deficit does not affect leaves' stomata opening and therefore does not have a significant effect on $\mathrm{P}_{\mathrm{n}}, g_{\mathrm{S}}, \mathrm{Ci}$, and $\mathrm{E}$. In the present study, $\mathrm{P}_{\mathrm{n}}$ and $g_{\mathrm{S}}$ were less affected under $\mathrm{W}_{1}$, and there was no significant difference with the GK. Severe drought stress significantly inhibited the photosynthesis of Eleuthero by limiting $\mathrm{CO}_{2}$ and causing photodamage in medicinal plants (Cornic and Massacci, 1996).

Changes in medicinal compound. In the present study, the UPLC-MS method was successfully applied for the quantitative analysis of six medicinal compounds in Eleuthero. The comprehensive score ( $\mathrm{Q}$ value) of principal component analysis was used to

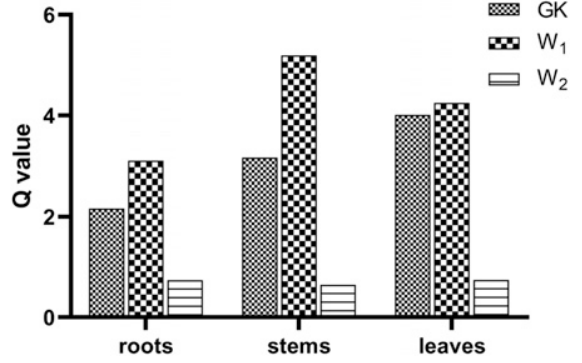

Fig. 5. Global effects of different water stress conditions on medicinal compounds accumulation in Eleuthero. The soil moisture of contro $(\mathrm{GK})$, moderate drought stress $\left(\mathrm{W}_{1}\right)$, and severe drought stress $\left(\mathrm{W}_{2}\right)$ groups was maintained at 0.8 to $0.9 \mathrm{~g} / \mathrm{g}, 0.5 \pm 0.05 \mathrm{~g} / \mathrm{g}$, and $0.3 \pm 0.05 \mathrm{~g} / \mathrm{g}$ respectively. $\mathrm{n}=6$.

illustrate the overall trend of the contents of the compounds studied in roots, stems, and leaves under different drought stress conditions (Fig. 5). The compounds studied showed the same accumulation trend in different organs. Higher content of the compounds studied was observed in $\mathrm{W}_{1}$. In the roots, the higher content of eleutheroside B, isofraxidin, kaempferol, and hyperoside accumulated in $\mathrm{W}_{1}(P<0.05)$. Among them, 
the contents of eleutheroside B and isofraxidin in GK were higher than those in $\mathrm{W}_{2}$, and the contents of kaempferol and hyperoside in $\mathrm{W}_{2}$ were higher than those in GK. The contents of rutin and eleutheroside $\mathrm{E}$ were higher in $\mathrm{GK}$ and the lower in $\mathrm{W}_{2}$. In stems, the contents of eleutheroside $\mathrm{B}$, isofraxidin, hyperoside, kaempferol, and rutin in $\mathrm{W}_{1}$ were significantly higher than those in other treatments $(P<0.05)$. Among them, the contents of eleutheroside B, isofraxidin, hyperoside, and kaempferol in GK were higher than those in $\mathrm{W}_{2}$, and the contents of rutin in $\mathrm{W}_{2}$ were higher than those in GK. The content of eleutheroside $\mathrm{E}$ in $\mathrm{GK}$ was higher than that in other treatment groups $(P<0.05)$. In the leaves, the contents of eleutheroside $\mathrm{B}$, isofraxidin, and eleutheroside $\mathrm{E}$ in $\mathrm{W}_{1}$ were significantly higher than those in other treatments $(P<0.05)$, and the contents of eleu- theroside $\mathrm{B}$ and eleutheroside $\mathrm{E}$ in $\mathrm{GK}$ were higher than those in $\mathrm{W}_{2}$. Isofraxidin was not detected in GK. The contents of rutin, kaempferol, and hyperoside were the highest in GK and the lowest in $\mathrm{W}_{2}$ (Fig. 6).

The results showed that the content of eleutheroside $\mathrm{B}$, eleutheroside $\mathrm{E}$, and isofraxidin was higher in roots and stems (Fig. 4AC), similar to previous research (Kang et al., 2001; Lee et al., 2005), and further confirmed
A

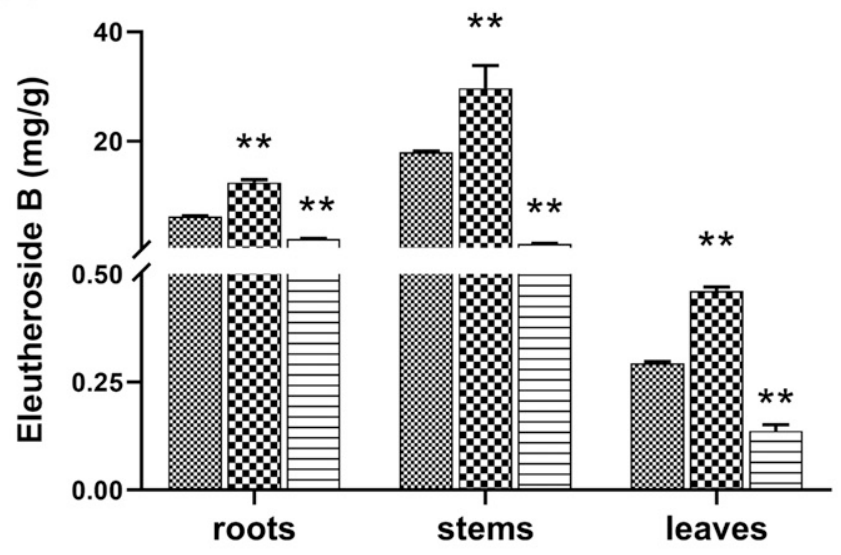

C

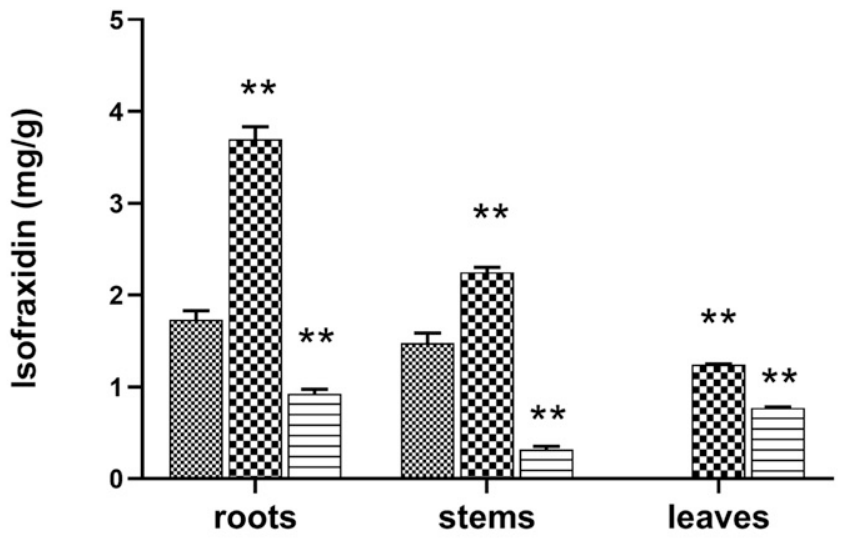

E

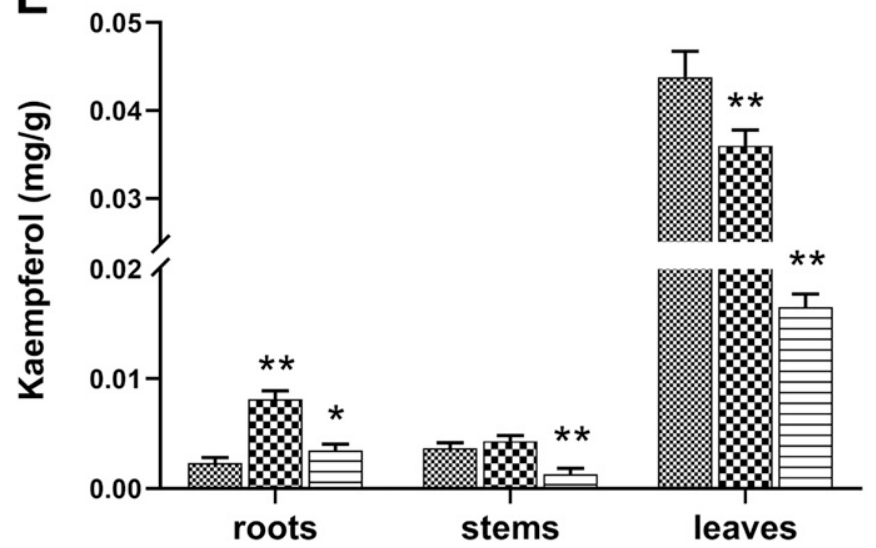

B

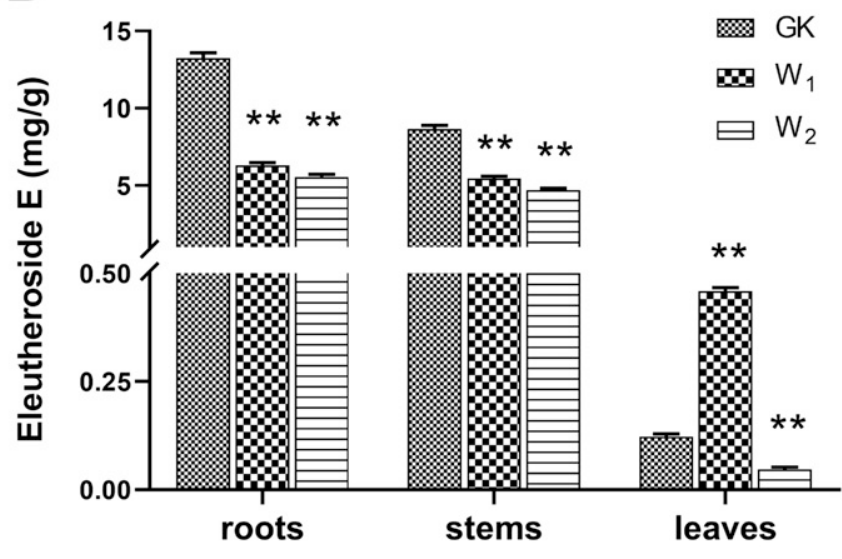

D

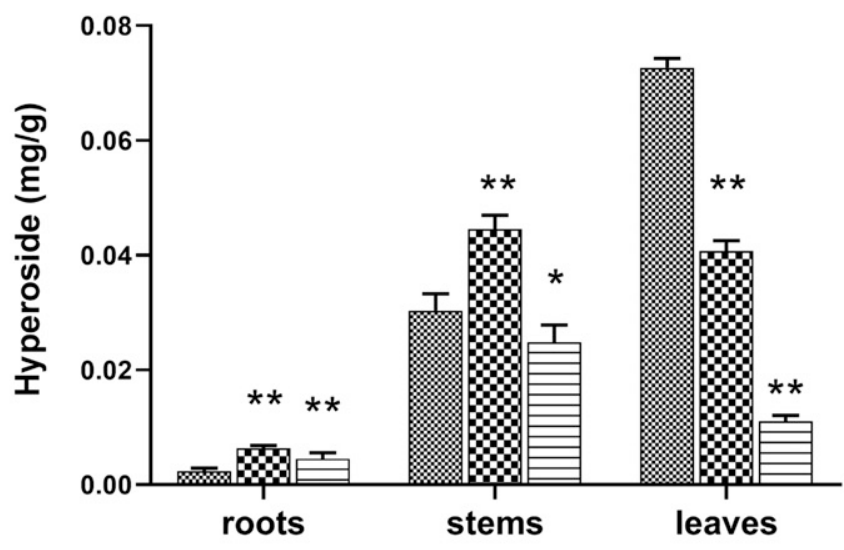

$\mathbf{F}$

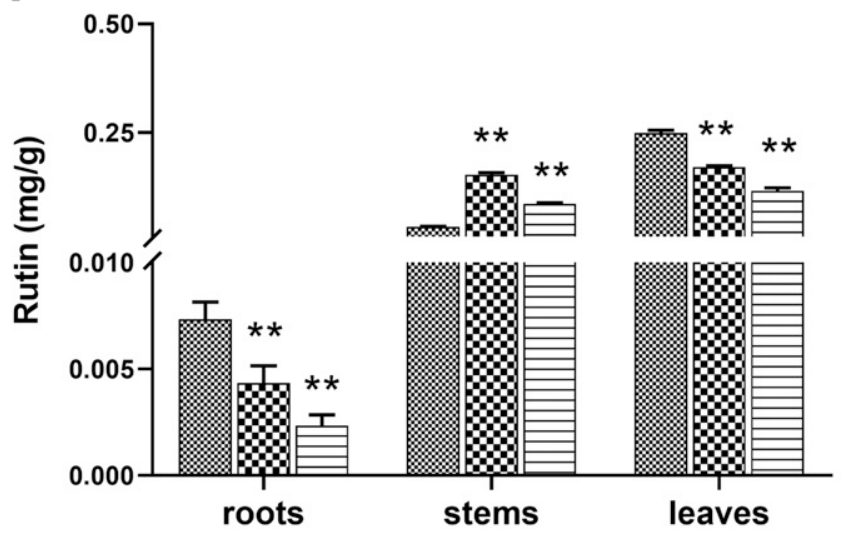

Fig. 6. Effects of different water stress on the accumulation of six medicinal compounds in Eleuthero roots. The soil moisture of control (GK), moderate drought stress $\left(\mathrm{W}_{1}\right)$, and severe drought stress $\left(\mathrm{W}_{2}\right)$ groups was maintained at 0.8 to $0.9 \mathrm{~g} / \mathrm{g}, 0.5 \pm 0.05 \mathrm{~g} / \mathrm{g}$, and $0.3 \pm 0.05 \mathrm{~g} / \mathrm{g}$ respectively. Bars indicate standard deviation $(\mathrm{n}=6) . * *$ Significant difference between the treatment group and the control group $(P<0.01)$; *significant difference between the treatment group and the control group $(P<0.05)$. 
the correctness of the Chinese Pharmacopoeia's roots and stems as the legal medicinal organs of Eleuthero. Eleutheroside B and isofraxidin showed the same accumulation trend in different organs under different drought stress conditions, and their content was higher in the $\mathrm{W}_{1}$ group (Fig. $4 \mathrm{~A}$ and $\mathrm{C}$ ), which showed that $\mathrm{W}_{1}$ is suitable for the accumulation of two compounds. Drought stress inhibited the accumulation of eleutheroside $\mathrm{E}$ in roots and stems. The content of eleutheroside $\mathrm{E}$ was higher in GK, indicating that the moist soil environment is suitable for the accumulation of this component. It should be pointed out that the content of eleutheroside B is the highest among the six compounds, which verified the validity of eleutheroside B as the quality control index of Eleuthero in the Chinese Pharmacopoeia. It has been shown that eleutheroside E possesses an antistress and antifatigue effect (Kimura and Sumiyoshi, 2004; Weng et al., 2007). The results of the present study indicate that the accumulation of eleutheroside $\mathrm{E}$ in different organs of Eleuthero was also high, which is in agreement with results of previous studies (Lee et al., 2005; Zhang and Xue, 2008). Eleutheroside $\mathrm{E}$ thus has the potential to become a quality control indicator for Eleuthero.

The results of the present study show a significant effect of hyperoside, rutin, and kaempferol (all flavonoids) in different parts of Eleuthero. The content of the three flavonoids was higher in the leaves of Eleuthero, and the content decreased with the soil drought stress. The preceding results indicate that a wet soil environment is suitable for the accumulation of flavonoids in Eleuthero. This is inconsistent with previous research on St. John's wort and Hypericum pruinatum (Caliskan et al., 2017; Gray et al., 2003). This could be explained by the fact that phenylpropanoids and shikimate pathway enzymes reduce their activity under drought stress, as has been demonstrated in other plants (Mewis et al., 2012; Sanchez-Rodriguez et al., 2011). Previous studies (He et al., 2019; Liu et al., 2019; Qian et al., 2019) have shown that these compounds have antiinfective, immunomodulatory, and other pharmacological effects. Our results show that Eleuthero leaves have good medicinal value. In this study, the content of hyperoside and kaempferol was low, and whether this relates to the growth period remains to be studied.

\section{Conclusions}

This investigation provided data on the impact of drought stress on the morphological and photosynthetic characteristics and the medicinal compounds of Eleuthero. The growth parameters results show that drought stress may inhibit plant growth, and moderate drought stress may significantly inhibit photosynthesis. The medicinal compounds were significantly affected in different parts of Eleuthero under various water treatments. Moderate drought stress is the most suitable condition for the accumulation of medicinal compounds in Eleuthero. This study may provide the experimental basis for further study on the metabolic regulation mechanisms of the medicinal components of Eleutherococcus senticosus under drought stress.

\section{Literature Cited}

Bączek, K., J.L. Przybył, O. Kosakowska, and Z. Wegglarz. 2017. Accumulation of phenolics in eleuthero (Eleutherococcus senticosus (Rupr. Et Maxim.) Maxim.) as affected by plant development. Acta Sci. Pol. Hortorum Cultus 16(4):89-99.

Becerra-Moreno, A., M. Redondo-Gil, J. Benavides, V. Nair, L. Cisneros-Zevallos, and D.A. Jacobo-Velazquez. 2015. Combined effect of water loss and wounding stress on gene activation of metabolic pathways associated with phenolic biosynthesis in carrot. Front. Plant Sci. 6:837.

Berry, J.A. and W.J.S. Downton. 1982. Environmental regulation of photosynthesis. Photosynthesis 2:263-343.

Bucci, L.R. 2000. Selected herbals and human exercise performance. Amer. J. Clin. Nutr. 72(2):624S-636S

Caliskan, O., J. Radusiene, K.E. Temizel, Z Staunis, C. Cirak, D. Kurt, and M.S. Odabas. 2017. The effects of salt and drought stress on phenolic accumulation in greenhouse-grown Hypericum pruinatum. Ital. J. Agron. 12:271275.

Chaves, M. 1991. Effects of water deficits on carbon assimilation. J. Expt. Bot. 42(1):1-16.

Cichello, S.A., Q. Yao, A. Dowell, B. Leury, and X.-Q. He. 2015. Proliferative and inhibitory activity of siberian ginseng (Eleutherococcus senticosus) extract on cancer cell lines; A-549, XWLC-05, HCT-116, CNE and Beas-2b. Asian Pac. J. Cancer Prev. 16(11):4781-4786.

Colom, M.R. and C. Vazzana. 2001. Drought stress effects on three cultivars of Eragrostis curvula: Photosynthesis and water relations. Plant Growth Regulat. 34(2): 195-202.

Cornic, G. and A. Massacci. 1996. Leaf photosynthesis under drought stress. Springer, Rotterdam, the Netherlands.

Dixon, R.A. and N.L. Paiva. 1995. Stress-induced phenylpropanoid metabolism. Plant Cell 7(7):1085-1097.

Farooq, M., A. Wahid, N. Kobayashi, D. Fujita, and S. Basra. 2009. Plant drought stress: Effects, mechanisms and management, $p$. 153-188. In: E. Lichtfouse, M. Navarrete, P. Debaeke, S. Véronique, and C. Alberola (eds.). Sustainable agriculture. Springer.

Fei, X.J., L.L. Zhu, L.M. Xia, W.B. Peng, and Q. Wang. 2014. Acanthopanax senticosus attenuates inflammation in lipopolysaccharide-induced acute lung injury by inhibiting the NF-kappaB pathway. Genet. Mol. Res. 13(4):10537-10544.

Ferreres, F., D.M. Pereira, P. Valentao, P.B Andrade, R.M. Seabra, and M. Sottomayor 2008. New phenolic compounds and antioxidant potential of Catharanthus roseus. J. Agr. Food Chem. 56(21):9967-9974.

Gamir, J., V. Pastor, A. Kaever, M. Cerezo, and V. Flors. 2014. Targeting novel chemical and constitutive primed metabolites against Plectosphaerella cucumerina. Plant J. 78(2):227240.

Garcia-Calderon, M., T. Pons-Ferrer, A. Mrazova, P. Pal'ove-Balang, M. Vilkova, C.M. PerezDelgado, J.M. Vega, A. Eliasova, M. Repcak, A.J. Marquez, and M. Betti. 2015. Modulation of phenolic metabolism under stress conditions in a Lotus japonicus mutant lacking plastidic glutamine synthetase. Front. Plant Sci. 6:760.

Gray, D., S. Pallardy, H. Garrett, and G. Rottinghaus. 2003. Effect of acute drought stress and time of harvest on phytochemistry and dry weight of St. John's wort leaves and flowers. Planta Med. 69(11):1024-1030.

Han, J., L.-Q. Bian, X.-J. Liu, and F. Zhang. 2014. Effects of Acanthopanax senticosus polysaccharide on signal transduction of peripheral lymphocyte of weaning piglets. Nat. Prod. Res Dev. 10:1589-1592.

Han, Y., A. Zhang, H. Sun, Y. Zhang, X. Meng, G. Yan, L. Liu, and X. Wang. 2017. High-throughput ultra high performance liquid chromatography combined with mass spectrometry approach for the rapid analysis and characterization of multiple constituents of the fruit of Acanthopanax senticosus (Rupr. et Maxim.) Harms. J. Sep. Sci. 40(10):2178-2187.

Harb, A., A. Krishnan, M.M. Ambavaram, and A. Pereira. 2010. Molecular and physiological analysis of drought stress in Arabidopsis reveals early responses leading to acclimation in plant growth. Plant Physiol. 154(3):1254 1271.

He, J.T., H.Q. Li, G.F. Li, and L. Yang. 2019. Hyperoside protects against cerebral ischemiareperfusion injury by alleviating oxidative stress, inflammation and apoptosis in rats. Biotechnol. Biotechnol. Equip. 33(1):798-806.

Huang, L., H. Zhao, B. Huang, C. Zheng, W. Peng, and L. Qin. 2011. Acanthopanax senticosus: Review of botany, chemistry and pharmacology. Pharmazie 66(2):83-97.

Jiang, W., W. Li, L. Han, L. Liu, Q. Zhang, S. Zhang, T. Nikaido, and K. Koike. 2006. Biologically active triterpenoid saponins from Acanthopanax senticosus. J. Nat. Prod. 69(11):1577-1581

Jiang, Y.-y. and M.-H. Wang. 2015. Different solvent fractions of Acanthopanax senticosus harms exert antioxidant and anti-inflammatory activities and inhibit the human Kv1. 3 channel. J. Med. Food 18(4):468-475.

Kang, J.S., P.T. Linh, X.F. Cai, H.S. Kim, J.J. Lee, and Y.H. Kim. 2001. Quantitative determination of eleutheroside B and E from Acanthopanax species by high performance liquid chromatography. Arch. Pharm. Res. 24(5):407-411.

Kim, Y.-H., M.L. Cho, D.-B. Kim, G.-H. Shin, J.-H. Lee, J.S. Lee, S.-O. Park, S.-J. Lee, H.M. Shin, and O.-H. Lee. 2015. The antioxidant activity and their major antioxidant compounds from Acanthopanax senticosus and A. koreanum. Molecules 20(7):13281-13295.

Kimura, Y. and M. Sumiyoshi. 2004. Effects of various Eleutherococcus senticosus cortex on swimming time, natural killer activity and corticosterone level in forced swimming stressed mice. J. Ethnopharmacol. 95(2-3):447-453.

Lee, S.-H., S.-S. Kang, S.-H. Cho, S.-N. Ryu, and B.-J. Lee. 2005. Determination of eleutherosides $\mathrm{B}$ and $\mathrm{E}$ in various parts of Acanthopanax species. Korean J. Pharmacogn. 36(2):70-74.

Lee, S., D. Son, J. Ryu, Y.S. Lee, S.H. Jung, J. Kang, S.Y. Lee, H.-S. Kim, and K.H. Shin. 2004. Anti-oxidant activities of Acanthopanax senticosus stems and their lignan components. Arch. Pharm. Res. 27(1):106-110.

Li, J.-L., N. Li, H.-S. Lee, S.-S. Xing, S.-Z. Qi, Z.-D. Tuo, L. Zhang, B.-B. Li, J.-G. Chen, and L. Cui. 2016. Four new sesqui-lignans isolated from Acanthopanax senticosus and their diacylglycerol acyltransferase (DGAT) inhibitory activity. Fitoterapia 109:185-189.

Liu, F., Y.H. Zhao, J.M. Lu, S.H. Chen, X.G Zhang, and W.W. Mao. 2019. Hyperoside 
inhibits proinflammatory cytokines in human lung epithelial cells infected with Mycoplasma pneumoniae. Mol. Cell. Biochem. 453(1-2): 179-186.

Mewis, I., M.A. Khan, E. Glawischnig, M. Schreiner, and C. Ulrichs. 2012. Water stress and aphid feeding differentially influence metabolite composition in Arabidopsis thaliana (L.). PLoS One 7(11):e48661, doi: 10.1371/ journal.pone.0048661.

Mustafa, N.R. and R. Verpoorte. 2007. Phenolic compounds in Catharanthus roseus. Phytochem. Rev. 6(2-3):243-258.

Pellegrino, A., E. Lebon, T. Simonneau, and J. Wery. 2010. Towards a simple indicator of water stress in grapevine (Vitis vinifera L.) based on the differential sensitivities of vegetative growth components. Austral. J. Grape Wine Res. 11(3):306-315.

Qian, J.C., X.M. Chen, X.J. Chen, C.C. Sun, Y.C. Jiang, Y.Y. Qian, Y.L. Zhang, Z.A. Khan, J.M. Zhou, G. Liang, and C. Zheng. 2019. Kaempferol reduces K63-linked polyubiquitination to inhibit nuclear factor-kappa B and inflammatory responses in acute lung injury in mice. Toxicol. Lett. 306:53-60.

Rajendrudu, G., C.V. Naidu, and K. Mallikarjuna. 2000. Effect of water stress on photosynthesis and growth in two teak phenotypes. Photosynthetica 36(4):627-630.

Sanchez-Rodriguez, E., D.A. Moreno, F. Ferreres, M. Rubio-Wilhelmi Mdel, and J.M. Ruiz. 2011.
Differential responses of five cherry tomato varieties to water stress: Changes on phenolic metabolites and related enzymes. Phytochemistry 72(8):723-729.

Schafellner, C., R. Berger, A. Dermutz, E. Führer, and J. Mattanovich. 1999. Relationship between foliar chemistry and susceptibility of Norway spruce (Pinaceae) to Pristiphora abietina (Hymenoptera: Tenthredinidae). Can. Entomol. 131(3):373-385.

Sun, H., J. Liu, A. Zhang, Y. Zhang, X. Meng, Y. Han, Y. Zhang, and X. Wang. 2016. Characterization of the multiple components of Acanthopanax senticosus stem by ultra high performance liquid chromatography with quadrupole time-of-flight tandem mass spectrometry. J. Sep. Sci. 39(3):496-502.

Van der Weele, C.M., W.G. Spollen, R.E. Sharp, and T.I. Baskin. 2000. Growth of Arabidopsis thaliana seedlings under water deficit studied by control of water potential in nutrient-agar media. J. Expt. Bot. 51(350):1555-1562.

Walton, D.C., M.A. Harrison, and P. Cotê. 1976. The effects of water stress on abscisic-acid levels and metabolism in roots of Phaseolus vulgaris L. and other plants. Planta 131(2):141-144.

Weng, S., J. Tang, G. Wang, X. Wang, and H. Wang. 2007. Comparison of the addition of Siberian ginseng (Acanthopanax senticosus) versus fluoxetine to lithium for the treatment of bipolar disorder in adolescents: A random- ized, double-blind trial. Curr. Ther. Res. Clin. Exp. 68(4):280-290.

Wu, K.X., J. Liu, Y. Liu, X.R. Guo, L.Q. Mu, X.H. $\mathrm{Hu}$, and Z.H. Tang. 2018. A comparative metabolomics analysis reveals the tissuespecific phenolic profiling in two Acanthopanax species. Molecules 23(8), doi: 10.3390/ molecules23082078.

Yamazaki, T. and T. Tokiwa. 2010. Isofraxidin, a coumarin component from Acanthopanax senticosus, inhibits matrix metalloproteinase-7 expression and cell invasion of human hepatoma cells. Biol. Pharm. Bull. 33(10):1716-1722.

Yang, F., L. Yang, W. Wang, Y. Liu, C. Zhao, and Y. $\mathrm{Zu}$. 2012. Enrichment and purification of syringin, eleutheroside $\mathrm{E}$ and isofraxidin from Acanthopanax senticosus by macroporous resin. Int. J. Mol. Sci. 13(7):8970-8986.

Yang, L., H. Ge, W. Wang, Y. Zu, F. Yang, C. Zhao, L. Zhang, and Y. Zhang. 2013. Development of sample preparation method for eleutheroside B and E analysis in Acanthopanax senticosus by ionic liquids-ultrasound based extraction and high-performance liquid chromatography detection. Food Chem. 141(3): 2426-2433.

Zhang, J. and Q. Xue. 2008. HPLC determination of syringin and eleutheroside $\mathrm{E}$ in different parts of Acanthopanax senticosusRupr. et Maxim. Harms. Chinese Journal of Pharmaceutical Analysis 28(12):2018-2020. 